\title{
PREVENTIVE PRESERVATION OF CULTURAL HERITAGE WITHIN A HOT AND HUMID CLIMATE: A CASE STUDY OF TAINAN CONFUCIAN TEMPLE, TAIWAN
}

\author{
YU CHIEH CHU, MIN FU HSU, TSAI YU LIN \& PEI YU YI \\ Conservation Science Section, Bureau of Cultural Heritage, Ministry of Culture, Taiwan
}

\begin{abstract}
We took the example of the Tainan Confucian Temple to explore preventive preservation of Taiwanese cultural assets within a hot and humid climate. We mainly used microclimate monitoring data and computational fluid dynamics simulation to analyze the overall environment and identify problem areas, which were shown by the results to be the Ritual Implement Storeroom and Musical Instrument Storeroom. The latter had the highest humidity due to a mango tree located at the rear with a canopy stretching 4-6 $\mathrm{m}$ across the roof, which blocked the sunlight during the day and prevented any reduction in humidity. This storeroom includes the original calligraphic wall panels and central beam of the Minglun Hall; therefore we propose measures to improve the current environment without modifying the building materials or structures. First, we suggest either changing the display items or shifting the exhibition area, and transplanting or pruning the mango tree. Second, dehumidification or ventilation equipment should be placed not only in the Musical Instrument Storeroom, but also in the Chung Sheng Shrine and Ta Cheng Palace at night during the hot season, and in the Ritual Appliance Storeroom in the day during the cold season, to improve environmental problems.

Keywords: historic building, preventive conservation, CFD, natural ventilation, micro climate.
\end{abstract}

\section{INTRODUCTION}

Climate change has become a critical issue worldwide. In 1992, the "United Nations Framework Convention on Climate Change" began to pay attention to this issue. In 2015, the Paris Agreement further stated goals backed by law that require mitigating or coping with climate change. Climate change will enlarge existing risks and produce new risks to nature and humans. In response to abnormal climate conditions, countries have turned their attention to preventive preservation of cultural heritage and initiated a series of plans and actions. The Noah's Ark Project, 2004-2007, of the Sixth Framework Program supported by the European Union, the Smart Monitoring of Historic Structures (2008-2010) of the Seventh Framework Program project, and the Climate for Culture (2009-2014) project all have the goal of preventing future climate and environmental change from affecting cultural heritage on both a macroscale and microscale. Similarly, the Tokyo National Research Institute for Cultural Properties has conducted numerous studies with the purpose of planning preventive preservation efforts, especially regarding outdoor cultural properties. They have adopted methods such as long-term monitoring or surveying, modelling experiments, and computational fluid dynamics (CFD) simulation analysis to analyze the relationship between preservation objects and the environment and to propose control strategies and improvement methods. Their research results have mainly yielded repair strategies.

Taiwan has a hot and humid climate, which is not ideal for preserving traditional buildings. As the climate changes, preservation tasks continue to become increasingly difficult, especially for historical buildings because they have been exposed to the external environment for long periods of time. If Taiwan does not act quickly, preservation and repair work will be even more costly than it is now. Compared with other countries that have taken 
efficacious actions in recent years, Taiwan has only just begun to act in this area. Preventive preservation in Taiwan requires further research and implementation. Therefore, this study selected a critical cultural heritage of Taiwan, collected basic environmental data in its vicinity, and applied these data to verify the CFD model. The model was then used to simulate the current environment, highlight problem areas, and provide improvement suggestions that do not involve changing the building material or structure.

\section{STUDY AREA}

The Tainan Confucius Temple (TCT) is the first Confucian temple in Taiwan. Located in Tainan City in southern Taiwan, the TCT was constructed in 1665. At that time, it was the only school that Taiwanese children could attend, and thus it is also referred to as the first school in Taiwan. In 1684, the TCT was expanded to its current scale. Because the TCT is a critical cultural asset of Taiwan, the government has declared it a national heritage site. Because of its high cultural value, this study used it as an example on which to base a discussion concerning the preventive preservation of a cultural asset located in an urban environment among a dense cluster of contemporary buildings. The simulation area encompassed a circle with a radius of $500 \mathrm{~m}$ with the temple at the center, covering the TCT buildings, surrounding buildings, a plantation, and a water pool.

The primary spaces of the major construction of the TCT all have openings that face the courtyard. The openings are the primary vertical plane and are made of wood. The other three walls are all made of brick. Ta Cheng Hall, the East and West Wings, and Chongsheng Shrine have their doors and windows open all day long. The Ritual Implements Storeroom, Musical Instrument Storeroom, and Yi Cheng School have their doors and windows closed at 17:30 each day. The Ritual Implements Storeroom and Musical Instrument Storeroom have small windows, and during the day, a double-panel door is kept open. Because of concern that the displays would be damaged by sunlight, the northern windows of the Musical Instrument Storeroom are kept closed all day. The inner side of the wall exhibits peeling paint. The floor is wet, generating efflorescence. In addition, the northwest side of the external wall of the Musical Instrument Storeroom leaks, and moss grows on the moist wall. A mango tree was planted here, covering a quarter of the roof of the Musical Instrument Storeroom. Regarding the external side of the northern wall, large areas of paint have peeled off, and moss and fungi prosper. Ta Cheng Hall has an elevated roof, and its doors and windows were open all day long. Yi Cheng School has two double-panel doors and one single-panel door open during the day. Chongsheng Shrine is an open space, so it is easily influenced by the external climate. Its floor is severely weathered and peeled.

\section{MATERIALS AND METHODS}

\subsection{CFD model}

Because the TCT is located amidst urban architecture, the CFD model we constructed focused on block and scale. The depiction of the TCT itself included a detailed model of its indoor and outdoor spaces. Because the slope of the roof would affect the simulation results, it was portrayed in detail. The existing plantation was surveyed, and the survey documented the breed and height of the trees as well as the width of the crowns. The survey results were used in the CFD model to reconstruct the plantation and to simulate the influence of the plantation on the surrounding environment. 


\subsection{CFD simulation}

CFD has been developed into a powerful assessment tool that is especially useful for assisting with the design of architecture and cities [1]. CFD provides the designer with precise parameters with which to plan urban or residential areas to improve the windy and thermal environment in the city, around buildings, or indoors. The implementation of CFD also reduces the effect of new designed projects on the surrounding environment and decreases future energy use. General research using CFD has studied ventilation in a building or a city, pedestrian wind fields, contaminant diffusion, the urban heat island effect, and wind energy [2]-[5].

This study used WindPerfect DX developed by E-Sim (Japan) for simulation. The governing equations were solved using LES. The simulation model was $1,000 \mathrm{~km}$ in diameter with the Confucius Temple at the center. The entire simulation area was 2,000 $\mathrm{m} \times 2,000 \mathrm{~m}$ $\times 450 \mathrm{~m}$ in dimensions with the Confucius Temple as the focused area, whose coverage was $350 \mathrm{~m} \times 350 \mathrm{~m}$. Because the street width was 3-6 m and the height of buildings in the Confucius Temple was 4-9 m, the grid dimensions of the focused area was $1.2 \mathrm{~m} \times 1.2 \mathrm{~m} \times$ $1.2 \mathrm{~m}$. The grid density decreased gradually from the focused area outward. The total number of grids was 9,580,889. According to the latest Wind Resistance Design Specifications and Commentary of Buildings published by the Construction and Planning Agency, Ministry of the Interior [6], this area is categorized as Locality A. The surface roughness is $\alpha=0.32$. Using this value, the vertical distribution of the average velocity at the section can be calculated using eqn (1)

$$
\frac{V}{V_{0}}=\left[\frac{Z}{z_{0}}\right]^{\alpha}
$$

where

- $\quad V$ : wind speed $(\mathrm{m} / \mathrm{s})$ at height $Z$;

- $Z_{0}$ : wind velocity at reference height $(\mathrm{m} / \mathrm{s})$;

- $\quad Z$ : a certain height $(\mathrm{m})$;

- $V_{0}$ : height of the meteorological station (m);

- $\alpha$ : exponent value.

\subsection{CFD validation}

The on-site data collected on the hottest day during the hot season in 2017 (13 September) were used to verify the CFD model. The maximum error of wind velocity, temperature, and humidity was no more than $1 \mathrm{~m} / \mathrm{s}, 0.2^{\circ} \mathrm{C}$, and $6 \%$, respectively. The aforementioned small gap between simulation values and observed values demonstrated that the CFD model had explanatory power.

\section{RESULTS AND DISCUSSION}

This study used the observed data to verify the CFD model, confirming that it was able to represent the on-site situation. Subsequently, the climate data from the past five years were used to simulate the thermal field, humidity field, and wind field of the TCT in the hot season and cold season as well as during the day and night, and the environmental problems affecting indoor and outdoor areas of the TCT were examined. 


\subsection{Daytime in the hot season}

During this period, the wind mainly comes from the west. The simulation results revealed that the wind field in the courtyard between the TCT and the semi-circular pond was optimal. Minglun Hall and Wen Chang Pavilion were densely surrounded by trees approximately 6-7 $\mathrm{m}$ tall and with crowns 4-5 $\mathrm{m}$ wide. Although the crowns were trimmed, because the trees were planted densely together, this region exhibited a large area of wind shadow (Fig. 1). In the courtyard, the wind velocity at the east and west sides of the Ta Cheng Hall increased (Fig. 2), which subsequently influenced the wind velocity inside the East and West Wings, creating an average wind velocity of $0.2-0.5 \mathrm{~m} / \mathrm{s}$ indoors. The indoor wind field of the East Wing was better than that of the West Wing. The Ritual Implements Storeroom and the Musical Instrument Storeroom had small windows, and thus the indoor wind velocity was lower, approximately $0.1 \mathrm{~m} / \mathrm{s}$ or slower.

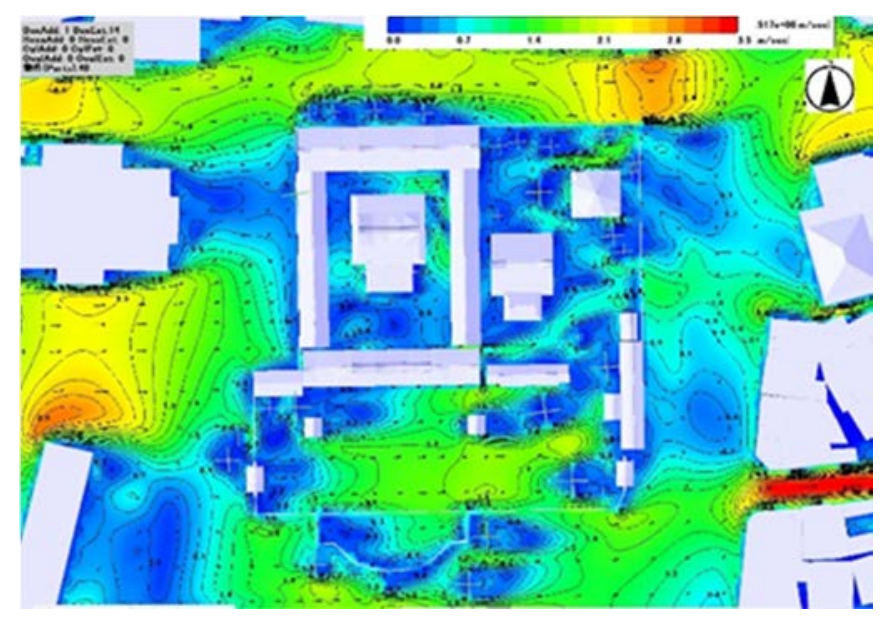

Figure 1: Wind field simulation of the outdoor space and the vicinity.

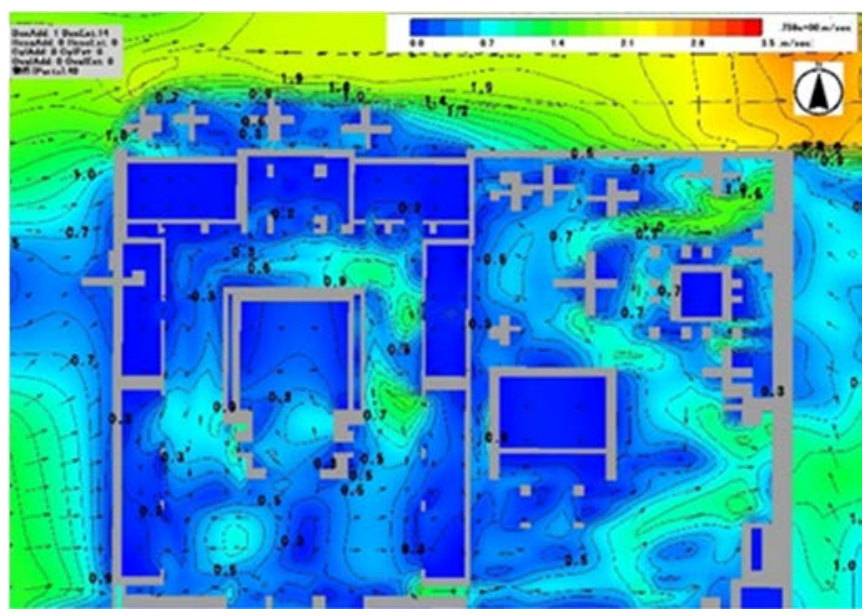

Figure 2: Indoor wind field simulation. 
The Musical Instrument Storeroom, as well as both ends of the front corridor of the Storehouse of Books and Records and Yi Cheng School, exhibited high humidity compared with other spaces (Fig. 3). The average relative humidity of the front corridor ends of Yi Cheng School and the Storehouse of Books and Records was $82.7 \%$ and $85.5-86.3 \%$, respectively. The average humidity of the Musical Instrument Storeroom was approximately $84.3-86.7 \%$. These are problematic areas with poor ventilation. Because their relative humidity was over $70 \%$, the humidity problem had to be addressed. We advise that the side doors at the end of the front corridor of the Storehouse of Books and Records and Yi Cheng School be opened regularly to facilitate ventilation, thereby reducing humidity. As for the Musical Instrument Storeroom, indoor ventilation facilities or dehumidifiers could be installed to actively dehumidify the space during the day in the hot season.

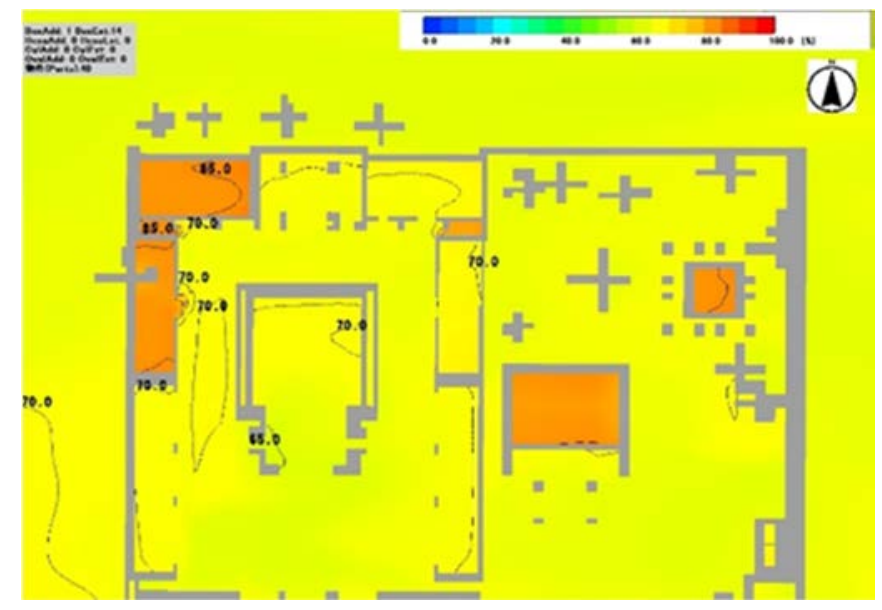

Figure 3: Indoor humidity field simulation.

\subsection{The night time in hot season}

During this period, the wind was mainly from the south. The outdoor relative humidity was over $80 \%$, and the average temperature was approximately $28.9-29.2^{\circ} \mathrm{C}$. The indoor simulation demonstrated that the indoor average temperature was approximately $29-30^{\circ} \mathrm{C}$, and the indoor humidity of all regions was higher than $83 \%$ (Fig. 4). The humidity of the Musical Instrument Storeroom was the highest; its average relative humidity was $92.2-99 \%$. We advise that a dehumidifier or ventilation equipment be installed in this place to mitigate the humidity problem at this time period during this season.

\subsection{The daytime in cool season}

The wind during this period mainly came from the north-northeast direction. The wind follows the street to the vicinity of the TCT, and the wind velocity increased. The wind velocity in the courtyard between the temple and the semi-circular pond was approximately $0.6-1.0 \mathrm{~m} / \mathrm{s}$. However, within the TCT fence, the courtyard surrounded by Ta Cheng Hall and Ta Cheng Gate was influenced by buildings and a dense plantation, and many wind shadows were formed. The wind velocity there was between 0.2 and $0.5 \mathrm{~m} / \mathrm{s}$, which is not conducive to ventilation. 


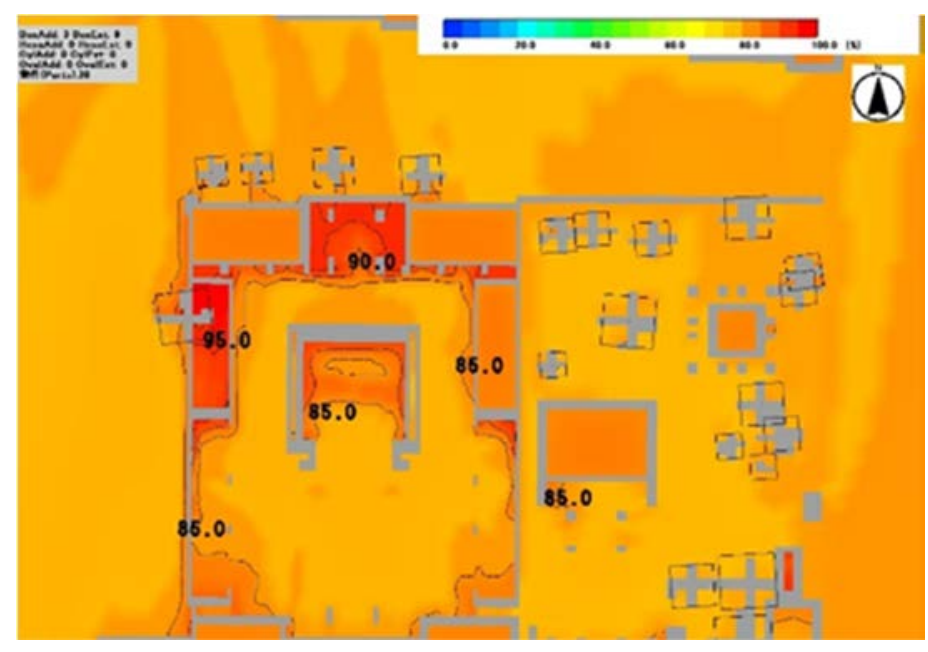

Figure 4: Indoor humidity field simulation.

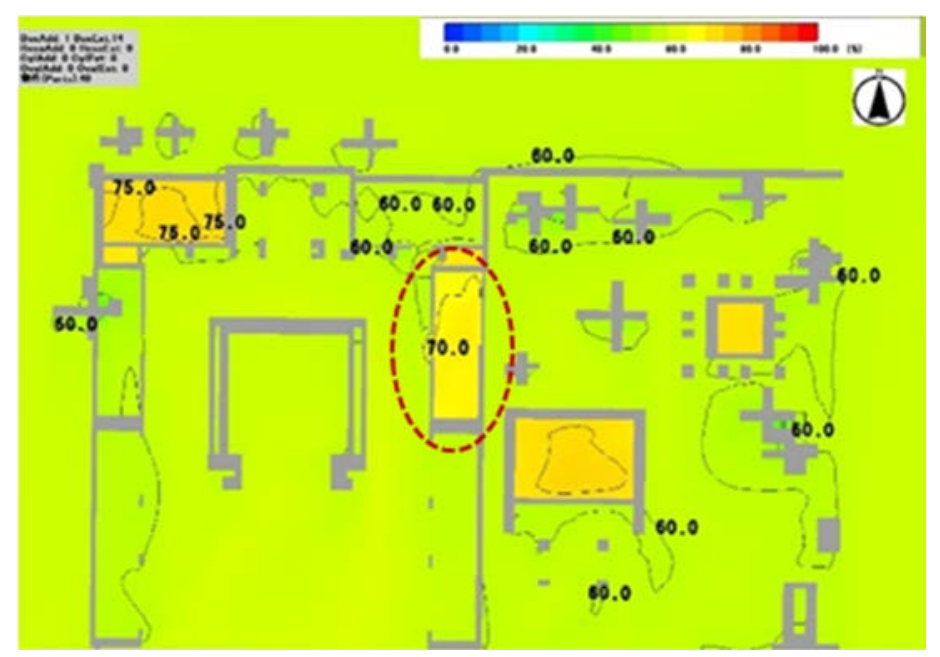

Figure 5: Ritual Implements Storeroom humidity distribution (marked by the red dashed line circle).

The indoor space simulation results indicated that except for spaces such as the Storehouse of Books and Records, which was closed all day long, at this time period during this season, the Ritual Implements Storeroom has the highest relative humidity (Fig. 5). Although the wind velocity at the east and west sides of Ta Cheng Hall increased, it only increased the indoor wind velocity of the East and West Wings to $0.3-0.5 \mathrm{~m} / \mathrm{s}$. The indoor wind velocity of the Ritual Implements Storeroom remained approximately $0.02-0.1 \mathrm{~m} / \mathrm{s}$. Because the indoor area has almost no wind, the Ritual Implements Storeroom should be dehumidified during that season or have increased ventilation to reduce humidity. 


\subsection{The night time in cool season}

The major wind direction was north-northeast. During the night time, the windows of the Ritual Implements Storeroom, Musical Instrument Storeroom, and Yi Cheng School were all shut, and only the windows at Ta Cheng Hall and the East and West Wing were open. Indoor spatial simulation results revealed that the humidity of the Musical Instrument Storeroom in the night time during the cool season was higher than that of other spaces, averaging $88-95 \%$ (Fig. 6). Additionally, its indoor temperature was approximately $20-23^{\circ} \mathrm{C}$, which facilitates the formation of dew. We suggest that this place conduct night time dehumidification or open the doors and windows to maintain ventilation to reduce humidity.

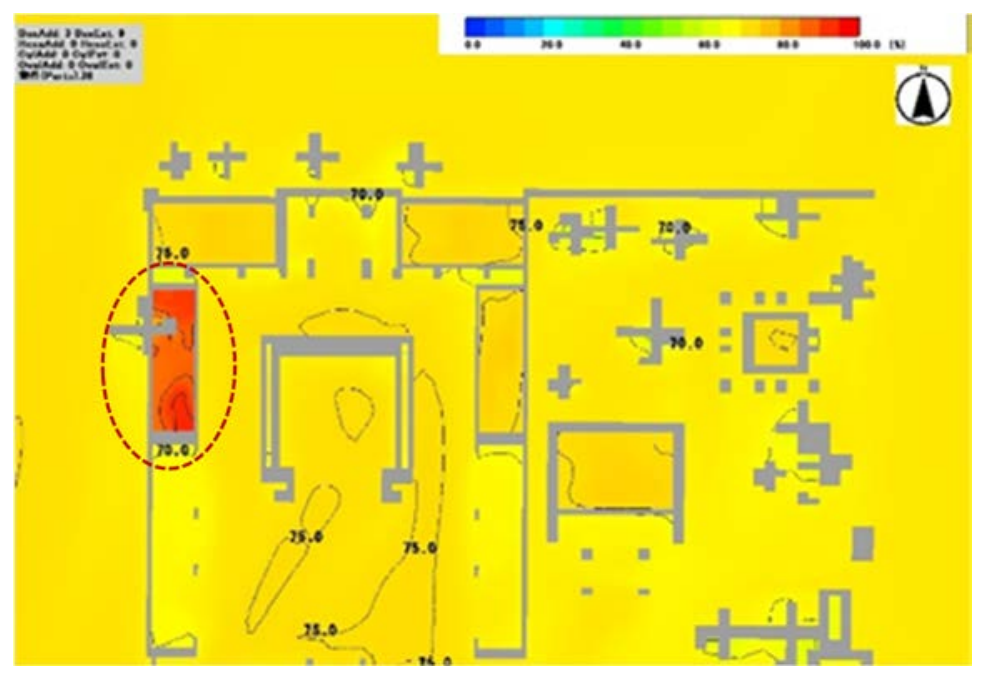

Figure 6: Indoor humidity field simulation (Musical Instrument Storeroom is marked with the red dashed line circle).

\section{CONCLUSION}

Preventive preservation jobs often use microclimate research to discover environmental problems, thereby enabling the preventive preservation of objects from damage or deterioration. Therefore, this study used on-site data and simulation to discover environmental problems. Simulation results indicated that the Ritual Implements Storeroom and the Musical Instrument Storeroom were the areas with major problems and required indoor ventilation and dehumidifiers. Specifically, the indoor space of the Musical Instrument Storeroom has the highest average relative humidity. Also, the mango tree with a crown 4-6 m wide shaded the roof during the day, blocking the sun and hindering the reduction of humidity in the room. Because the exhibits of that area are the original sculptured internal wall and the middle beam of Minglun Hall, we suggest altering the exhibit content or transplanting or trimming the mango tree. In addition, during the evening of the hot season, the relative humidity of Ta Cheng Hall and Chongsheng Shrine were over 80\%, for which we suggest proactive dehumidification. The ends of the front corridor of Yi Cheng School and the Storehouse of Books and Records had relative humidity over $80 \%$ during the day in the hot season, and we suggest that side doors be opened during that time to increase ventilation and reduce humidity. 


\section{REFERENCES}

[1] Blocken, B., van der Houtc, A., Dekkerd, J. \& Weilerc, O., CFD simulation of wind flow over natural complex terrain: Case study with validation by field measurements for Ria de Ferrol, Galicia, Spain. Journal of Wind Engineering and Industrial Aerodynamics, 147, pp. 43-57, 2015.

[2] Chu, Y.C., Hsu, M.F. \& Hsieh, C.M., An example of ecological wisdom in historical settlement: The wind environment of Huazhai Village in Taiwan. Journal of Asian Architecture and Building Engineering, 16(3), pp. 463-470, 2017.

[3] Chu, Y.C., Hsu, M.F. \& Hsieh, C.M., The impacts of site selection and planning of a historic settlement on a sustainable residence. Applied Ecology and Environmental Research, 15(2), pp. 145-145, 2017.

[4] Mochida, A. \& Lun, I.Y.F., Prediction of wind environment and thermal comfort at pedestrian level in urban area. Journal of Wind Engineering and Industrial Aerodynamics, 96(10-11), pp. 1498-1527, 2008.

[5] Amorim, J.H., Rodrigues, V., Tavares, R., Valente, J. \& Borrego, C., CFD modelling of the aerodynamic effect of trees on urban air pollution dispersion. Science of The Total Environment, 461-462, pp. 541-551, 2013.

[6] Construction and Planning Agency, Ministry of the Interior, Taiwan, Wind Resistance Building Design Specifications and Commentary of Buildings, 109(2), 2014. 